Recepción: 04/ 03/ 2016

Aceptación: 15 / 06/ 2016

Publicación: 06/ 01/2017

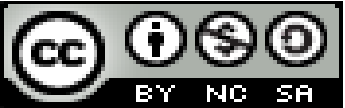

Ciencias de la educación

Artículo de investigación

\title{
Una mirada sobre la acreditación en América Latina
}

A look at accreditation in Latin America

Um olhar sobre a acreditação na América Latina

Jahaira Alexandra García-Aráuz ${ }^{1}$

jahairitagarcia@hotmail.com

Correspondencia: jahairitagarcia@hotmail.com

${ }^{1}$ Magister Universidad Laica Eloy Alfaro de Manabí, Manta, Ecuador 


\title{
Resumen
}

Teniendo en cuenta que todo proceso de formación le corresponde la búsqueda de niveles superiores de calidad que propicien su eficiencia, eficacia, pertinencia e idoneidad entre otros aspectos, así como la necesidad de que los actores de estos tengan plena conciencia de las fortalezas y debilidades que les impidan o favorezcan el alcance de parámetros de calidad cada vez más elevados aceptados por la comunidad académica, profesional y empresarial. A tales efectos se realizó una revisión bibliográfica exhaustiva donde se analizan algunos aspectos con respecto a la acreditación universitaria en América Latina.

Palabras clave: Acreditación; evaluación; calidad; programas.

\begin{abstract}
Taking into account that all training processes are responsible for the search for higher levels of quality that promote their efficiency, effectiveness, relevance and suitability among other aspects, as well as the need for the stakeholders to be fully aware of the strengths and weaknesses that Impede or favor the achievement of ever higher quality parameters accepted by the academic, professional and business community. To that end, a comprehensive bibliographic review was carried out, where some aspects regarding university accreditation in Latin America
\end{abstract}

Key words: Accreditation; evaluation; quality; programs.

\section{Resumo}

Dado que todo o processo de formação corresponde à busca de níveis de qualidade mais elevados que promovem a eficiência, eficácia, relevância e adequação entre outros aspectos, bem como a necessidade de os actores destes são plenamente consciente dos pontos fortes e fracos impedi-los ou favorecer a gama de parâmetros de cada vez maior qualidade aceite pela comunidade acadêmica, profissional e empresarial. Para este efeito, uma ampla revisão da literatura, onde as análises foi realizada alguns aspectos com relação à acreditação universitária na América Latina.

Palavras chave: Acreditação; avaliação; qualidade; programas. 


\section{Introducción}

La idea de universidad, aunque sin ese nombre aún, nace con quienes crearon la cultura griega, ligados entre sí solo por su amor al saber racional, crítico y analítico. Sus meditaciones sobre la importancia social y política de aquel, y la influencia creciente del sabio en el poder los llevó a considerar que tenían un deber para con el Estado y la sociedad. (Centro Interuniversitario de Desarrollo. 1993).

La importancia del conocimiento como factor fundamental de cultura y progreso del hombre y la sociedad adquiere un reconocimiento creciente, en particular cuando se crean en Europa, las primeras universidades, corporaciones de profesores y alumnos sobre las que recae desde entonces, en forma primordial, la responsabilidad de la creación y transmisión de la cultura, así como la de la formación y capacitación de pensadores, artistas, investigadores y profesionales. (Centro Interuniversitario de Desarrollo. 1993).

Las universidades nacen en Hispanoamérica en el siglo XVI con el fin de capacitar a sus estudiantes para el servicio de la Iglesia y del Estado. Su organización siguió el modelo de las universidades de Ávila y Salamanca y su metodología docente era eminentemente expositiva. El maestro transmitía el conocimiento a sus alumnos desde su cátedra o tarima. (Centro Interuniversitario de Desarrollo. 1993).

En la actualidad, los países de América Latina están empeñados en un intenso proceso de desarrollo social y de crecimiento económico, que exige la utilización efectiva de todos sus recursos, en especial de la capacidad científica y tecnológica nacional, tanto para la selección, adecuación y transferencia de tecnologías adecuadas desde el exterior, así como para la creación de tecnologías propias. (Centro Interuniversitario de Desarrollo. 1993).

La interdependencia es una de las marcas más características de la actual globalización. La mayoría de los fenómenos locales y nacionales están en buena parte influenciados por fenómenos internacionales y, muchas veces, universales (Días Sobrinho, 2005). A problemas y tendencias universales también pueden corresponder problemas y respuestas nacionales distintas y específicos. Las demandas planteadas a la educación superior son hoy muy amplias y exigen respuestas diversificadas en cuanto a tipos de organización y funciones institucionales. Un 
análisis de las políticas de acreditación en América Latina y el Caribe tiene que enfrentar, aunque con brevedad, el tema de la diferenciación, complejidad y heterogeneidad de los sistemas e instituciones de educación superior en las distintas realidades nacionales. Esto tiene que ver con la difícil pero fundamental cuestión de asegurar la calidad en contextos asimétrico. (Dias Sobrinho, 2009).

Así mismo, no existe consenso sobre la noción de Calidad. Para evitar fragmentaciones dispensadoras e inoperantes, la solución mundialmente aceptada consiste en atribuir a entidades u organismos acreditados la prerrogativa de elaborar lineamientos generales, objetivos y propuestas operacionales sobre calidad. Esto facilita la comprensión y las prácticas de acreditación, pero también representa un desplazamiento parcial de la autonomía desde las universidades hacia los organismos de acreditación. (Dias Sobrinho, 2009).

\section{Evolución de la Educación Superior Latinoamericana}

Fernández Lamarra (2012), en su estudio plantea que la educación superior latinoamericana ha registrado fuertes incrementos en la segunda mitad del siglo XX. Así el número de instituciones universitarias pasó de 75 en 1950 a más de 2.000 aproximadamente en la actualidad, en su gran mayoría privadas. El número de estudiantes pasó de 276.000 en 1950 a alrededor de 18 millones actualmente. Es decir que la matrícula se multiplicó por más de 60 veces en 60 años.

La tasa de incremento anual de la matrícula desde 1990 ha sido del orden del 6\%. Esta tasa ha sido mucho mayor para la universidad privada (8\%) que para la pública $(2,5 \%)$. Esto indica que actualmente alrededor del 60\% - quizás más - de la matrícula universitaria en América Latina concurre a universidades privadas, a diferencia de lo que ocurría hasta la década del 80, en que la universidad latinoamericana era predominantemente estatal. El extremo mayor lo constituye Brasil con un $85 \%$ de matrícula privada en la educación superior. Países como Colombia y Chile están alrededor del $60 \%$ de matrícula privada y México - a pesar de su importante tradición de educación pública a lo largo de gran parte del siglo XX - tiene una matriculación privada en la educación superior del orden del 45\%. Los países con menor matrícula privada son Cuba con 0\%, Uruguay con el 10\% y Argentina con alrededor del 20\%. (Fernández Lamarra, N. 2012). 
En los últimos años la educación superior en América Latina ha tenido un cambio sustancial marcado por el nacimiento de los sistemas de acreditación y evaluación de la calidad. En apenas pocos años casi todos los países han creado por ley en el marco de complejos procesos de discusiones, estas agencias de supervisión de la calidad, que no sólo han significado nuevas modalidades de regulación de la educación terciaria, sino también la colocación de la calidad de la educación como el centro de las Universidades. (Fernández Lamarra, N. 2012).

\section{Antecedentes acreditación universitaria}

A partir de los años 70, varios países de América Latina ya introducen el tema de la calidad en sus propuestas de reforma de la educación superior. Sin embargo, el tema de la evaluación sólo entra en la agenda de los países a mediados de los 90, como consecuencia de dos fenómenos complementarios: por una parte, el incremento de la demanda por educación superior, y por otra, el desarrollo de un sector privado de corte empresarial. El incremento de la demanda está asociado al éxito de los programas de desarrollo de la educación primaria y secundaria, junto con la complejización creciente del sector productivo. Pero inevitablemente, esta mayor demanda significó cambiar el modelo selectivo y elitista de la educación superior latinoamericana por una perspectiva más inclusiva, asociada a una población estudiantil de menores recursos económicos y culturales. Al mismo tiempo, la incorporación de estos nuevos proveedores privados generó en la mayoría de los países un fuerte cuestionamiento a la legitimidad y calidad de partes importantes de la oferta educativa superior. Más tardíamente, a principios de la década en curso, surge el tema de la acreditación como una preocupación generalizada en toda la región, incluso en el Caribe. Lemaitre (2007) lo apunta como una "consecuencia de la centralidad que ha adquirido la educación superior en el marco de lo que solemos llamar la sociedad del conocimiento, o la era de la información, y su rol como componente esencial del desarrollo de los países".

En América Latina, se enfatiza la importancia de desarrollar modelos nacionales y centrar los procesos de aseguramiento de la calidad en agencias también nacionales. Sin embargo, no se puede desconocer que este énfasis nacional se asocia fuertemente a la búsqueda de arreglos integradores, en los cuales las agencias nacionales se comprometen con ciertos objetivos regionales con el fin de llegar a procesos de reconocimiento mutuo. Es el caso, por ejemplo, del 
MERCOSUR, donde mediante un acuerdo sobre estándares y procedimientos se llegó a acuerdos de reconocimiento mutuo de las carreras acreditadas; en Centroamérica, junto con fomentar la creación y desarrollo de agencias nacionales, se están creando agencias especializadas regionales, todas ellas - las nacionales y las regionales - acreditadas por un organismo regional. (Lemaitre,M.J. y Atria, JT. 2007).

Es innegable que hasta hoy no se ha desarrollado o aún no se ha consolidado una tradición de evaluación y de acreditación en muchos países de la región, a pesar de los excelentes programas de evaluación y de acreditación en países o sectores específicos. Si bien se puede afirmar que la consolidación de los modelos y experiencias de evaluación y acreditación es muy diversa, en general se observa una fuerte tendencia hacia la opción por sistemas de aseguramiento de la calidad complejos, que involucran procesos de licenciamiento (o autorización de nuevas instituciones), de evaluación, de acreditación o de auditoría académica. Estos procesos se aplican a nuevas instituciones o carreras, a estudiantes, a programas, a instituciones en una amplia gama que hace que la experiencia latinoamericana sea digna de un análisis cuidadoso.

La acreditación universitaria es el resultado de un proceso de evaluación y seguimiento sistemático y voluntario del cumplimiento de las funciones universitarias de una institución de educación superior (IES), que permite obtener información fidedigna y objetiva sobre la calidad de las instituciones y programas universitarios que desarrolla. Permite certificar ante la sociedad, la calidad de los recursos humanos formados y de los diferentes procesos que tienen lugar en una institución educativa. Es el reconocimiento formal y público otorgado a una institución académica en virtud del grado en que dicha institución o dentro de ésta, una o más de sus unidades, carreras o programas han logrado avances significativos en el cumplimiento de su misión y objetivos declarados, y satisface un conjunto acordado de criterios, indicadores y estándares de pertinencia y calidad. Descansa en la búsqueda permanente de la excelencia y representa el esfuerzo colectivo de la comunidad universitaria para rendir cuentas a sí misma y a la sociedad, sobre la pertinencia, relevancia y calidad de su ser y quehacer institucional. (Borroto Cruz R, Salas Perea R. 2004).

El propósito central de la acreditación es promover y estimular el continuo mejoramiento y determinar si una institución académica posee calidad a nivel general o respecto de una o más de 
sus carreras o programas educativos, si es capaz de demostrar que progresa de manera continua y sistemática, con el empleo de estrategias, procedimientos y recursos adecuados para el logro de su misión y sus objetivos, cumpliendo razonablemente con los criterios y normas de calidad establecidos. (Borroto Cruz R, Salas Perea R. 2004).

La acreditación no es permanente, sino que se otorga por un periodo que puede variar entre 5 y 10 años, a partir del cual puede ser renovada o retirada, sobre la base de los resultados obtenidos en el proceso de revisión o verificación efectuado. (Borroto Cruz R, Salas Perea R. 2004).

Consecuentemente, la acreditación universitaria se concibe como un mecanismo mediante el cual, la comunidad educativa establece y mantiene su autorregulación, y garantiza a los usuarios directos e indirectos de los servicios que ofrece, su integridad, pertinencia y calidad, en niveles que la hagan merecedora de la confianza y el respeto público . (Borroto Cruz R, Salas Perea R. 2004).

La gran meta de la universidad latinoamericana es responder a una triple misión:

- Hacer socialmente relevante su capacidad de creación cultural, de innovación científica y tecnológica y de formación de recursos humanos.

- Extender, por equidad, los conocimientos generados y acumulados en la institución a sectores amplios de la sociedad.

- Asegurar la excelencia por el mantenimiento y la mejora de la calidad de la enseñanza, investigación y extensión.

La evaluación institucional es, primordialmente, la valoración del nivel de calidad o excelencia de un centro educativo, pero a la vez, la calidad es además de objeto de la evaluación, meta, fin y hasta criterio de la propia evaluación, es decir, la calidad es el modelo organizado de la propia evaluación. (Borroto Cruz R, Salas Perea R. 2004).

Se consideran 2 dimensiones o enfoques de la calidad de la educación: 
- Satisfacción del educando o la familia como usuario o consumidor externo y satisfacción del trabajador (profesor, ejecutivo, administrador) como consumidor interno.

- Características del producto y su ajuste a determinados estándares predeterminados. Naturaleza de la educación, integridad de todos los factores del desarrollo humano, orden y coherencia en la relación lógica entre los factores que la componen y la eficacia del cumplimiento de sus funciones en cada uno de los procesos universitarios. (Borroto Cruz R, Salas Perea R. 2004).

Ambas dimensiones son necesarias y complementarias; la primera insiste en el para quién (a quien satisface), y la segunda en el qué y cómo (qué es la buena educación). La calidad en la educación es el efecto resultante de una relación peculiar entre los componentes básicos, internos y externos del sistema o del centro educativo. Al respecto se consideran los estándares de calidad siguientes:

- $\quad$ De tipo científico: validez, confiabilidad, generalizabilidad y objetividad.

- De tipo práctico-social: pertinencia, importancia, suficiencia, credibilidad, oportunidad, amplitud de difusión. (Borroto Cruz R, Salas Perea R. 2004).

Es importante la evaluación tanto de los resultados como de los procesos en sí mismos.

\section{Beneficios de la acreditación}

- Se obtiene un reconocimiento público de que la Universidad alcanzó parámetros de calidad destacables.

- Beneficia a todos los integrantes de la USS (alumnos, académicos y funcionarios) ya que consolida el prestigio y nos impulsa a avanzar en la mejora continua.

- El principal beneficio para los ex alumnos es que la acreditación garantiza que la Universidad entrega una formación profesional de calidad.

- En la acreditación institucional, una Universidad Acreditada permite a sus estudiantes optar a beneficios estatales, como becas, crédito con aval del estado, entre otros. 


\section{Experiencias de los procesos de acreditación}

Las experiencias cumplidas en América Latina y el Caribe y en Europa son recientes y han ganado centralidad, complejidad y diferenciación en los últimos años, en los que se asiste al surgimiento del concepto de aseguramiento (mejor dicho: garantía) de la calidad de la educación superior. (Chiancone Castro A 2005).

\section{Cuba}

El Ministerio de Educación Superior (MES) de la República de Cuba, desde su creación en 1976, le ha prestado gran importancia al control del trabajo de las instituciones de educación superior, como vía fundamental para lograr un mejoramiento continuo de la calidad. Dentro del sistema de control establecido se encuentra la evaluación institucional, la cual se ha ido perfeccionando y ajustando, tanto al nivel de desarrollo alcanzado por las instituciones de educación superior, como a los métodos y procedimientos empleados para ejercer la actividad de control.

Con respecto a la experiencias en evaluación y acreditación de programas de maestrías hasta enero /2015 se desarrollaron 31 procesos de evaluación externa a 21 programas de maestría con resultados satisfactorios, de los cuales 17 conservan vigencia de acreditación: 10 (58,8\%) de Excelencia, $6(35,3 \%)$ Certificados y $1(5,9 \%)$ Ratificado. Los resultados orientan hacia una adecuada utilización del sistema de gestión, concluyéndose la necesidad de fortalecer la cultura de autoevaluación. (Cunill López, M E. 2016).

\section{Chile}

La acreditación de las universidades en Chile se ha instalado paulatinamente, alcanzando un nivel de desarrollo relevante desde 2003, para llegar actualmente (2010) a contar con un $73 \%$ de instituciones acreditadas, y un $17 \%$ que no lo logró, un $2 \%$ que está en proceso, y un $8 \%$ de las universidades no han sido sometidas a esta instancia, por no cumplir con los requisitos legales para ello.

El alto grado de vinculación entre indicadores universitarios tradicionales e indicadores de acreditación, revela la necesidad de contar con sistemas de información públicos más complejos 
que permitan contar con antecedentes institucionales relevantes, de manera de disponer de estándares de comparación (benchmarking) conducentes al fortalecimiento de los procesos evaluativos, en beneficio de la mejora continua de las universidades y del sistema de educación superior chileno. (Cancino C 2014).

\section{Ecuador}

Art. 173.- Evaluación Interna, Externa, Acreditación, Categorización y Aseguramiento de la Calidad.- El Consejo de Evaluación, Acreditación y Aseguramiento de la Calidad de la Educación Superior normará la autoevaluación institucional, y ejecutará los procesos de evaluación externa, acreditación, clasificación académica y el aseguramiento de la calidad.

Las universidades, escuelas politécnicas, institutos superiores técnicos, tecnológicos, pedagógicos, de artes y conservatorios superiores del país, tanto públicos como particulares, sus carreras y programas, deberán someterse en forma obligatoria a la evaluación interna y externa, a la acreditación, a la clasificación académica y al aseguramiento de la calidad.

En septiembre de 2015, el Consejo de Evaluación, Acreditación y Aseguramiento de la Calidad de la Educación Superior -CEAACES- inició en un proceso de evaluación, acreditación y re categorización institucional en el que participación 13 Instituciones de Educación Superior -IES-.

Del total de universidades evaluadas, 12 participaron en la re categorización de forma voluntaria y solo para la Universidad de las Fuerzas Armadas (ESPE) el proceso fue obligatorio porque no fue incluida en la valoración del 2013. (Resultados de la acreditación y categorización vigentes. 2016)

Se concluye que la calidad es, actualmente, el problema central de la universidad latinoamericana y supone la consideración de la teoría y práctica de la evaluación, como uno de los elementos básicos para asegurarla. La evaluación no es una moda pasajera de la política de desarrollo institucional y científico, sino una herramienta de planificación y política universitaria que se aprecia será permanente. 


\section{Referencias bibliográficas}

Borroto Cruz R, Salas Perea R. (2004). Acreditación y evaluación universitarias Educ Med Sup; 200418 (3). Recuperado de http://bvs.sld.cu/revistas/ems/vol18_3_04/ems01304.htm

Chiancone Castro A, Martínez Larrechea E. (2005). Evaluación y Acreditación en la Educación Superior: Un estudio comparado de América Latina y de Europa. Recuperado de www.saece.org.ar/docs/congreso1/Martinez_evaluacion.doc

Cunill López, M E, Oramas González, R, Márquez Morales, N. (2016). Experiencias en evaluación y acreditación de programas de maestrías. Educación Médica Superior, 30(2) Recuperado de http://scielo.sld.cu/scielo.php?script=sci_arttext\&pid=S086421412016000200006\&lng=es\&tlng=es.

Cancino C, V, Schmal S, R. (2014). Sistema de Acreditación Universitaria en Chile: ¿Cuánto hemos avanzado? Estudios pedagógicos (Valdivia), 40(1), 41-60. Recuperado de https://dx.doi.org/10.4067/S0718-07052014000100003

Resultados de la acreditación y categorización vigentes.(2016). Consejo de evaluación, acreditación y aseguramiento de la calidad educación superior . Recuperado de http://www.ceaaces.gob.ec/sitio/acreditacion-y-categorizacion/

Centro Interuniversitario de Desarrollo. (1993). Acreditación Universitaria En América Latina Antecedentes y experiencias. $\quad$ Recuperado de https://www.cinda.cl/download/libros/Acreditaci\%C3\%B3n\%20Universitaria\%20en\%20Am\%C 3\%A9rica\%20Latina.\%20Antecedentes\%20y\%20Experiencias.pdf

Dias Sobrinho, José (2005). Dilemas da Educação Superior no mundo globalizado.

Sociedade do conhecimento ou economia do conhecimento? Casa do Psicólogo, São Paulo: 2005. 
Dias Sobrinho, José (2009). Acreditación de la educación superior en América latina y el Caribe. Recuperado de de https://upcommons.upc.edu/bitstream/handle/2099/7538/18_282295.pdf;jsessionid=77A775315FBBE81 A11C1E4CFA4A91578? sequence $=1$

Fernández Lamarra, N. (2012). Universidad y calidad en América Latina en perspectiva comparada: Interrogantes y desafíos. Avaliação: Revista da Avaliação da Educação Superior (Campinas), 17(3), 661-668. Recuperado de https://dx.doi.org/10.1590/S141440772012000300006

Lemaitre, M.J. y Atria, JT. (2007). Antecedentes para la legibilidad de títulos en países latinoamericanos, UNESCO-IESALC. 